\title{
Training in Robotic General Surgery: The Next Challenge
}

\section{Nicolas C Buchs}

Clinic for Visceral and Transplantation Surgery, Department of Surgery, University Hospital of Geneva, Switzerland

For more than 10 years now, robotic surgery has gained an increasing acceptance in all the surgical specialties. The interest has grown rapidly and the experience has increased with success, especially for advanced and complex procedures, such as hepatobiliary surgery, cardiac revascularization, valvular replacement, or difficult genitourinary resections for cancer. The new generation of surgeons is faced to this technological ( $\mathrm{r}-$ ) evolution. While it is clear that robotics is called to last in surgery, new challenges have emerged such as the training and the education of the next generation of residents and fellows.

For the first time in surgery, the question of training and teaching is not anymore a problem of person or personality but a problem of machine and device. For many years, training programs in robotics have been initiated all over the world for already trained surgeons. We have developed since 2006 our own robotic training program and more than 100 participants have followed the courses. Many of them have started a robotic program when they were back to their hospital. Moreover, the importance of proctorship has to be mentioned, at least for the very first cases. It allows the beginning of a safe and successful robotic program.

However, for novice residents, there are no real official guidelines to suggest minimum requirement during their residency. For example in USA, robotic surgery is not requested by Residency Review Committee [1]. Yet, it is true that this technology is expensive, especially with the da Vinci Si double console (Intuitive Surgical, Sunnyvale, CA) particularly dedicated for the training. It is even unrealistic for a majority of centers that cannot afford this investment. Though, we remain convinced that the double console is part of the solution, increasing the safety, while the senior surgeon keeps the control of the robot during a procedure performed by a resident. In fact, the training of the residents remains an open question. While it seems logical to develop this aspect, the residents have to proceed along with basic open and laparoscopic skills at the same time. With the time limitation, the days of work are not anymore endless. New solutions have to be developed and applied. For example, the role of simulators has to be emphasized. It is a simple and relatively cheap mean to bridge the gap between initial acquisitions of surgical skills and effective performance in a clinical setting using the robot. Like other centers in the USA [1], we started to expose our residents to robotic surgery in the operative room. In urology, the training seems well more in advance than in other specialties. Several groups [2-4] developed their own curriculum with encouraging results. It has been shown that a residency robotic program can be instituted without sacrificing the operative time, the surgical outcomes and the cost. Yet, different steps during this curriculum can be advised: live cases observation, simulation, dry laboratory, wet laboratory (animal, cadavers), patient-side assistance, and finally console side surgeon $[1,5]$ for basic procedures.

Finally, the need to start a robotic surgery training program as part of the residency curriculum in general surgery is urgent. While for already trained surgeons, all the facilities exist, for the residents there is an empty space. Integrating robotics during their curriculum is mandatory and will allow a safe development of this unique technology. Interestingly, if we search on Pubmed for "teaching and robotic surgery", we can discover 342 results, while for "technique" or "outcomes and robotic surgery" we can find more than 1500 results. It shows clearly that so far the interest of the surgical community is predominant in the technical aspect, but not in the education. Though, as staff surgeons, this is our mission to develop robotic surgery and to create a clear curriculum for the next generation.

\section{Acknowledgments}

I would like to thank all the robotic team at the University Hospital of Geneva Switzerland, particularly François Pugin, MD, Francesco Volonté, MD, and professor Philippe Morel, MD.

\section{References}

1. Bianco FM, Buchs NC, Coratti A, Kinzer K, Murphey M, et al. (2011) Training and education in robotic general surgery. Robotic Surgery 1: 16-23.

2. Grover S, Tan GY, Srivastava A, Leung RA, Tewari AK (2010) Residency training program paradigms for teaching robotic surgical skills to urology residents. Curr Urol Rep 11: 87-92.

3. Rashid HH, Leung YY, Rashid MJ, Oleyourryk G, Valvo JR, et al. (2006) Robotic surgical education: a systematic approach to training urology residents to perform robotic-assisted laparoscopic radical prostatectomy. Urology 68: 7579.

4. Huettner F, Dynda D, Ryan M, Doubet J, Crawford DL (2010) Robotic-assisted minimally invasive surgery; a useful tool in resident training--the Peoria experience, 2002-2009. Int J Med Robot 6: 386-393.

5. Chitwood WR Jr, Nifong LW, Chapman WH, Felger JE, Bailey BM, et al. (2001) Robotic surgical training in an academic institution. Ann Surg 234: 475-484.
Corresponding author: Nicolas C. Buchs, Clinic for Visceral and Transplantation Surgery, Department of Surgery University Hospital of Geneva, Rue GabrielPerret-Gentil, 41211 Geneva 14, Switzerland, E-mail: Nicolas.c.buchs@hcuge.ch

Received March 19, 2012; Accepted March 21, 2012; Published March 23, 2012

Citation: Buchs NC (2012) Training in Robotic General Surgery: The Next Challenge. J Adv Robot Automat 1:e104. doi:10.4172/2168-9695.1000e104

Copyright: @ 2012 Buchs NC. This is an open-access article distributed unde the terms of the Creative Commons Attribution License, which permits unrestricted use, distribution, and reproduction in any medium, provided the original author and source are credited. 